\title{
Value of computed tomography evaluation in pathologic classification and prognosis prediction of gastric neuroendocrine tumors
}

\author{
Shida Yan ${ }^{1 \#}$, Tongtong Liu ${ }^{2 \#}$, Ying $\mathrm{Li}^{3}$, Yongjian $\mathrm{Zhu}^{3}$, Jun Jiang ${ }^{3}$, Liming Jiang ${ }^{3}$, Hong Zhao ${ }^{1}$ \\ ${ }^{1}$ Department of Hepatobiliary Surgery, National Cancer Center, Cancer Hospital, Chinese Academy of Medical Sciences and Peking Union Medical \\ College, Beijing 100021, China; ${ }^{2}$ Department of Radiology, Beijing Chao-Yang Hospital, Capital Medical University, Beijing 100020, China; \\ ${ }^{3}$ Department of Radiology, National Cancer Center, Cancer Hospital, Chinese Academy of Medical Sciences and Peking Union Medical College, \\ Beijing 100021, China \\ Contributions: (I) Conception and design: L Jiang, H Zhao; (II) Administrative support: L Jiang; (III) Provision of study materials or patients: Y Li, \\ Y Zhu, J Jiang; (IV) Collection and assembly of data: S Yan, T Liu; (V) Data analysis and interpretation: S Yan, T Liu; (VI) Manuscript writing: All \\ authors; (VII) Final approval of manuscript: All authors. \\ \#These authors contributed equally to this work. \\ Correspondence to: Hong Zhao. Department of Hepatobiliary Surgery, National Cancer Center/Cancer Hospital, Chinese Academy of Medical \\ Sciences and Peking Union Medical College, No. 17 Panjiayuan Nanli, Chaoyang District, Beijing 100021, China. Email: zhaohong@cicams. \\ ac.cn; Liming Jiang. Department of Radiology, National Cancer Center/Cancer Hospital, Chinese Academy of Medical Sciences and Peking Union \\ Medical College, No. 17 Panjiayuan Nanli, Chaoyang District, Beijing 100021, China. Email: dr_jiangliming@126.com.
}

Background: The study aims to investigate the correlation of CT characteristics with pathological classifications and the prognostic value of CT features in patients with gastric neuroendocrine neoplasms (g-NENs).

Methods: Ninety-one cases of pathologically diagnosed g-NENs, including 15 cases of well-differentiated neuroendocrine tumors (NETs) (G1 and G2) and 76 cases of poor-differentiated neuroendocrine carcinomas (NECs) (G3 and MANEC) were retrospectively studied. All cases were included in correlation analysis of CT characteristics with pathologic grades. Among them, 76 patients who had fulfilled follow-up data were included for overall survival (OS) and disease-free survival (DFS) analysis.

Results: CT characteristics that favor poor differentiation include tumor location (fundus and cardia), larger tumor size $(>3.0 \mathrm{~cm})$, infiltrative growth, unclear tumor margin, serosa involvement, ulceration and lymph node metastasis $(\mathrm{P}<0.05)$. Most variables had sensitivities $>80 \%$ and specificities $>60 \%$ to distinguish NECs from NETs. Through log-rank analysis, it was revealed that serosa involvement, cystic degeneration, necrosis, heterogeneous enhancement and lymph node metastasis led to worse DFS and OS for patients with g-NENs $(\mathrm{P}<0.05)$. COX regression analysis showed that serosa involvement and lymph node metastasis were independent risk factor for DFS and OS, respectively, despite of grading, staging and therapeutic choices $(\mathrm{P}<0.05)$. Moreover, high Ki-67 index $(>55 \%)$ in G3 g-NENs is in correlation with serosa involvement and lymph node metastasis; accordingly, patients with higher Ki-67 index had worse 1-year DFS (61.7\% vs. $92.3 \% ; \mathrm{P}<0.05)$.

Conclusions: CT characteristics can be useful discriminators and prognostic factors for g-NENs and may help identify G3 g-NEC from G3 g-NEN by revealing its poor differentiation and high invasive potential.

Keywords: Stomach neoplasms; neuroendocrine tumors (NETs); multidetector computed tomography (CT); pathology; survival analysis

Submitted Jul 06, 2019. Accepted for publication Sep 10, 2019.

doi: $10.21037 /$ atm.2019.09.114

View this article at: http://dx.doi.org/10.21037/atm.2019.09.114 


\section{Introduction}

Gastric neuroendocrine neoplasms (g-NENs) are a heterogeneous group of tumors that are located at stomach and arise from cells of the diffuse neuroendocrine system. Once called as "carcinoid", it is currently defined as "neuroendocrine tumor" based on 2010 WHO classification of tumours of the digestive system (1) due to its properties of hormones and amines secretion and its malignant potential which further classifies it into three tiers: G1, G2 and G3. It is of clinical importance to discriminate the grade as it will affect the management strategy and prognosis (2). Furthermore, recent opinions suggest that G3 gastroenteropancreatic (GEP) NENs should be subdivided into G3 neuroendocrine tumor (NET) and G3 neuroendocrine carcinomas (NEC) (3), which has been applied in the classification of pancreatic NENs (4). The update is based on the findings that high-grade GEPNENs are heterogeneous diseases with diverse genetic and biological behaviors, as well as different therapeutic options and prognosis. The more specific and clear classification reveals its importance to clinical practices and treatment benefits. Therefore, it is necessary to further depict characteristics of NEN G3 from diverse dimensions (5), including CT imaging characteristics.

According to data from the Surveillance, Epidemiology, and End Results (SEER) program, the incidence rate of GEP-NETs is 3.56 per 100,000 in 2012 and the gastric NETs had the greatest rise in incidence with a 15 -fold increment from 1973 to 2012, possibly due to the increased diagnosis of patients with asymptomatic and early-stage disease by widely use of endoscopic procedures (6). In China, the stomach is one of the most common primary sites of GEP-NET (20.7-27.0\%), which has a higher proportion than those in western countries $(7,8)$. Prognosis of G-NENs is relatively satisfying as the 1-year overall survival (OS) rate $>80 \%$ and the 5 -year OS rate $>50 \%$, based on SEER data $(9,10)$; data from Chinese population shows no major difference $(8,11)$.

Computed tomography (CT) has been the standard imaging technique for complete staging and systematic evaluation of patients with g-NENs $(12,13)$. Although the preoperative pathological grading mainly depends on endoscopic biopsy, a small specimen from biopsy might cause misdiagnosis due to the nature of heterogeneity in $\mathrm{g}$-NEN; therefore, CT still has its value of assisting diagnosis. However, the CT characteristics of $g-N E N s$ have not been thoroughly investigated, especially the relationship between CT features and pathologic grades, as well as the predictive value of CT features on prognosis. In our study, we retrospectively analyzed the CT features of 98 cases of $\mathrm{g}$-NENs treated in our hospital to discovery the role of CT imaging in distinguishing different pathological grades and predicting patients' clinical outcomes.

\section{Methods}

\section{Patients}

Between January 2010 and January 2017, a total of 98 patients with g-NENs treated in Cancer Hospital, Chinese Academy of Medical Sciences were collected in our study. Patients who fulfilled the following inclusion criteria were included: (I) initial treatments and preoperative enhanced CT scanning were performed in our hospital; (II) the surgical operations were performed in our hospital and the postoperative pathological diagnoses were gastric NETs, which were graded according to the 2010 WHO classification of tumors of the digestive system; (III) for patients who received neoadjuvant treatments, the pathological diagnoses based on preoperative biopsies and surgical specimens should coincide; (IV) patients' clinical and image data should be detailed and intact; and $(\mathrm{V})$ patients whose primary gastric lesions should be detectable on CT images. Besides, patients (I) who received chemotherapies, radiotherapies or other cancerrelated treatments before CT scanning and (II) whose gastric contents were too much or gastric lesions were too small, which affected observation and measurement, were excluded. Among them, 7 patients who didn't receive surgery were excluded and 15 patients were lost to followup, left 76 cases for survival analysis. For each patient, the demographic information, clinical symptom, location of lesions, pathological characteristics, CT features, TNM stage, type of surgery, neoadjuvant and adjuvant treatment choice, recurrence-free survival and OS were recorded and analyzed. The study was approved by the institutional review board at our medical center.

\section{CT technique}

All patients underwent GE Highspeed MDCT with 64 detector-rows 6-8 hours after fasting. To achieve gastric distention, $500-800 \mathrm{~mL}$ of water was ingested before the examination. After unenhanced CT was performed, the patient would be injected nonionic contrast agent $(300 \mathrm{mgI} / \mathrm{mL}$ ) at a dose of $1.5 \mathrm{~mL} / \mathrm{kg}$ and a speed of $3.0-4.0 \mathrm{~mL} / \mathrm{s}$ by high pressure injector through the cubital 
vein. Then after $30-35$ and $60-65$ s, CT images in the arterial phase and the portal venous phase were collected respectively. The scan range was from the diaphragmatic dome to the line between the anterior superior iliac spines, with the section thickness of $0.5 \mathrm{~mm}$. However, due to the retrospective design of this study, most patients didn't receive standard enhanced multiple-phase scans. Among 91 patients, only 1 patient had intact dual-phase and unenhanced scans, while 18 only underwent dual-phase scans without unenhanced scans, 49 only had images in the portal venous phase, 12 only had images in the arterial phase and the rest 11 patients didn't receive enhanced scans.

\section{Image analysis}

Two senior radiologists (14 and 26 years of experience in abdominal CT, respectively) performed the image analysis. They were blinded to the gastroscopic results, lesion size, macroscopic features and stage of g-NENs. Difference in assessment were asked to be reevaluated by the chief radiologist with over 30 years of experience in abdominal $\mathrm{CT}$ and agreement was made with their consensus. The following parameters were assessed and recorded: lesion location, lesion size, growth pattern, boundary, invasion of the serosa layer, intratumoral areas of cystic change or necrosis, ulceration, adjacent organ involvement, enhancement pattern, lymph node and distant metastasis. The definitions of these features are described below.

For masslike tumors, the lesion size was recorded as the maximal diameter in the axial plane; while for tumors that grew infiltratively, the length of the involved stomach wall was recorded as the lesion size. The boundary was described as clear or unclear. The growth pattern was described as either infiltrative type or circumscribed type. The serosa layer wasn't considered to be invaded only if it was intact and has clear boundary with surrounding tissues, otherwise it was invaded. A lesion was considered to show areas of cystic change or necrosis if it contained a non-enhancing water-density area. The ulceration was considered when the gastric mucosa was discontinuous, had irregular morphology or had local depression. The enhancement pattern was described as homogeneous enhancement or inhomogeneous enhancement. A nodular soft-tissue lesion larger than $10 \mathrm{~mm}$ in short-axis diameter was considered as lymph node metastasis, referring to previous studies $(14,15)$ and our clinical experience. By comparing to pathological $\mathrm{N}$ stage, the diagnostic sensitivity and specificity of lymph node metastasis by CT was $89.5 \%$ and $81.8 \%$, respectively.
The representative CT images with descriptions are shown in Figures $S 1$ and $S 2$ in the supplementary material.

\section{Pathological classification}

The 2010 WHO classification (1) divides NETs into three histopathologic subtypes: NETs, NECs, and mixed adenoneuroendocrine carcinomas (MANECs). NETs are composed of well-differentiated cells with similar features of normal gastrointestinal endocrine cells and express neuroendocrine markers and hormones. According to ENETS consensus of grading proposal for foregut NETs (16), NETs have two tier, G1 and G2, based on mitotic count and Ki-67 index: G1, mitotic count $<2$ per $10 \mathrm{HPF}$ and/or $\leq 2 \% \mathrm{Ki}-67$ index; and G2, mitotic count 2-20 per $10 \mathrm{HPF}$ and/or $3-20 \% \mathrm{Ki}-67$ index. In the meantime, NECs belong to G3, defined as mitotic count $>20$ per $10 \mathrm{HPF}$ and/or $>20 \% \mathrm{Ki}-67$ index. Moreover, NECs encompass small cell and large cell types, which both are poorly differentiated and highly malignant neoplasm, with highly cellular atypia, extensive necrosis and higher mitotic figures. MANECs are complexes of adenocarcinoma and NET or NEC, and by arbitrary definition, each part should comprise at least $30 \%$ of the whole neoplasm.

\section{Statistical analysis}

Among demographic and clinical information, continuous variables were assessed using Mann-Whitney $U$ test while categorical variables were compared by $\chi^{2}$ test or Fisher's exact test. Among imaging parameters, continuous variables were compared using non-parametric Mann-Whitney $U$ test and $\chi^{2}$ test or Fisher's exact test was used for categorical variables. The OS was described using the Kaplan-Meier method. Univariate survival analysis was performed by the log-rank test. Multivariate survival analysis was performed by the COX regression model. Receiver operating characteristic (ROC) analysis was performed to determine the cutoff values and the sensitivity and specificity were calculated. Two-sided $\mathrm{P}$ values were computed and $\mathrm{P}<0.050$ was considered statistically significant. All aforementioned statistical analyses were implemented using SPSS version 22.0 for Windows (SPSS Inc., Chicago, IL, USA).

\section{Results}

\section{General characteristics and clinical presentations}

Among the 91 patients, there were 57 males and 34 females, 
with a M:F ratio of $1.68: 1$. The median age was 60 years old (range, 28-83 years). Based on the 2010 WHO pathological grading classification, 15 cases were confirmed as g-NETs (13 cases of G1 and 2 cases of G2), 54 cases were G3 $\mathrm{g}-\mathrm{NECs}$ and 22 were MANECs. Due to the unawareness of neuroendocrine neoplasms before, all patients didn't perform the test for gastrin and somatostatin receptor imaging, therefore classifying clinical subtypes was unable to fulfill. However, according to clinical classification from the ENETS guideline of gastroduodenal neoplasms (17), based on the fact of the absent of atrophic gastritis under endoscope and solitary lesion in all patients, we deemed they were all type III. For TNM staging, the majority belonged to stage III $(58,63.7 \%)$ and most patients with g-NETs were early stage (13 out of 15). Eighty-two cases (90.1\%) showed tumor-related clinical symptoms. The most common symptom was dysphagia $(36,39.6 \%)$, the remain common symptoms included abdominal pain $(28,30.8 \%)$, black stool (12, 13.2\%), abdominal distention (10, 11.0\%), vomit (4, 4.4\%) and regurgitation (4, 4.4\%). The rest 9 who didn't have any clinical manifestation included 7 patients with g-NETs and 2 patients with g-NECs, which showed statistically difference among the correlation between clinical symptoms and pathological types $(\mathrm{P}<0.001)$.

All patients underwent operations, including 62 radical subtotal gastrectomies (1 patient also had its hepatic metastasis resected), 9 radical total gastrectomies, 7 endoscopic submucosal dissections (ESD), 5 endoscopic mucosal resections (EMR) and 1 partial gastrectomy. Nearly all cases had R0 resections (86, 94.5\%). After operations, almost $70 \%$ received adjuvant chemotherapy and regimens for small cell lung cancer, including EP (etoposide + cisplatin) and EC (etoposide + carboplatin) were mostly applied. Besides, 12 patients received neoadjuvant treatments, which involved 10 with neoadjuvant platinumbased chemotherapy, 2 with somatostatin analog (octreotide). The detailed clinical characteristics were listed in Table 1.

\section{Correlations between CT features and pathological grades}

To discuss the predictive role of CT features for pathological diagnosis, patients with g-NECs or g-MANECs were compared with patients with g-NETs. The detailed variables involved in the analysis and results were listed in Table 2. Through the analyses using Chisquare test or Fisher exact test, we discovered that compared to g-NETs, g-NECs or g-MANECs would more likely to be found in the fundus and cardia, have larger size, grow infiltratively, have unclear tumor margin, have serosa involved, have ulceration, have heterogeneous enhancement and have metastatic lymph nodes detected in the CT images $(\mathrm{P}<0.001)$. However, variables including cystic degeneration and necrosis, surrounding organ invasion and distant organ metastasis found in the CT didn't show distinctly difference between two groups $(\mathrm{P}>0.05)$.

Besides, in regard to the tumor size, we measure the maximum diameter in the axial views and it showed that patients with g-NETs had the average tumor size of $2.31 \pm 1.54 \mathrm{~cm}$ while the other group's average tumor size was $4.38 \pm 1.74 \mathrm{~cm}(\mathrm{P}<0.001)$. ROC curve analysis and Youden index calculation manifested the tumor size of $3.0 \mathrm{~cm}$ was the best cutoff point, with an area under curve (AUC) of 0.836. Using it to distinguish g-NETs with g-NECs and g-MANECs had the sensitivity of $76.3 \%$ and the specificity of $80.0 \%$. We also calculated the sensitivities and specificities of other variables that significantly differed $\mathrm{g}$-NETs with g-NECs and g-MANECs and listed in the Table 3. Most variables had sensitivities over $80 \%$ and specificities over $60 \%$. The growth pattern (localized or infiltrative) had the highest sensitivity (98.7\%) and the serosa involvement had the highest specificity (93.3\%).

Moreover, patients with $\mathrm{g}-\mathrm{NEC}(\mathrm{N}=47,7$ patients without Ki-67 data), which were diagnosed based on 2010 WHO classification, were subdivided into two groups according to the Ki-67 index. The cut-off point was chosen to be $55 \%$ followed by previous literatures $(3,4)$. Interestingly, by comparing imaging characteristics between two subgroups, serosa involvement and lymph node metastasis were proved to be significantly more frequent in G3 g-NENs with Ki-67 index >55\% (Table 4; $\mathrm{P}<0.05$ ).

\section{CT features and survival analysis}

Among 76 patients who received surgeries and had intact follow-up data (Table 5), most of them had lesions in the fundus and cardia $(44,57.9 \%)$, while the majority were TNM stage III $(49,64.5 \%)$ and pathologically diagnosed as NECs or MANECs $(62,81.6 \%)$. Towards tumor sizes, the average maximum diameter of the lesion in the axial plane was $4.03 \mathrm{~cm}$ (range, $0.8-8.0 \mathrm{~cm}$ ). Moreover, since $3.0 \mathrm{~cm}$ was the best cutoff point to differentiate G3 NEC from G1/2 NET (mentioned previously), we still used it as the cutoff point for survival analysis and found most cases had tumors $\geq 3.0 \mathrm{~cm}(51,67.1 \%)$. Towards other imaging characteristics, most lesions grew infiltratively (68, 89.5\%) 
Table 1 Clinicopathological characteristics of 91 cases of gastric neuroendocrine neoplasms

\begin{tabular}{|c|c|}
\hline Variables & No. (\%) \\
\hline Age, mean [range] & 60 [28-83] \\
\hline \multicolumn{2}{|l|}{ Gender } \\
\hline Male & $57(62.6)$ \\
\hline Female & $34(37.4)$ \\
\hline \multicolumn{2}{|l|}{ Clinical symptom } \\
\hline Dysphagia & $36(39.6)$ \\
\hline Abdominal pain & $28(30.8)$ \\
\hline Black stool & $12(13.2)$ \\
\hline Abdominal distention & $10(11.0)$ \\
\hline Vomit & $4(4.4)$ \\
\hline Regurgitation & $4(4.4)$ \\
\hline None & $9(9.9)$ \\
\hline \multicolumn{2}{|l|}{ TNM stage } \\
\hline I & $13(14.3)$ \\
\hline II & $15(16.5)$ \\
\hline III & $58(63.7)$ \\
\hline IV & $4(4.4)$ \\
\hline Unknown & $1(1.1)$ \\
\hline \multicolumn{2}{|l|}{ Pathological pattern } \\
\hline G1 & $13(14.3)$ \\
\hline G2 & $2(2.2)$ \\
\hline G3 & $54(59.3)$ \\
\hline MANEC & $22(24.2)$ \\
\hline \multicolumn{2}{|l|}{ Operation } \\
\hline Radical subtotal gastrectomy & $61(67.0)$ \\
\hline Proximal & $47(51.6)$ \\
\hline Distal & $14(15.4)$ \\
\hline $\begin{array}{l}\text { Distal \& hepatic metastasis } \\
\text { resection }\end{array}$ & $1(1.1)$ \\
\hline Radical total gastrectomy & $9(9.9)$ \\
\hline Partial gastrectomy & $1(1.1)$ \\
\hline $\begin{array}{l}\text { Endoscopic submucosal } \\
\text { dissection }\end{array}$ & $7(7.7)$ \\
\hline Endoscopic mucosal resection & $5(5.5)$ \\
\hline Unknown & $7(7.7)$ \\
\hline
\end{tabular}

Table 1 (continued)
Table 1 (continued)

\begin{tabular}{lc}
\hline Variables & No. (\%) \\
\hline Resection margin & $86(94.5)$ \\
R0 & $1(1.1)$ \\
R1 & $4(4.4)$ \\
R2 & \\
Neoadjuvant treatment & $10(11.0)$ \\
Neoadjuvant chemotherapy & $2(2.2)$ \\
Somatostatin analog & $79(86.8)$ \\
None & \\
Adjuvant chemotherapy & $63(69.2)$ \\
Yes & $21(23.1)$ \\
No & $7(7.7)$ \\
Unknown
\end{tabular}

and had unclear margins $(65,85.5 \%)$; in the meantime, they mostly had serosa involved $(47,61.8 \%)$, heterogenous enhancement $(54,79.4 \%)$, ulceration $(53,69.7 \%)$ and metastatic lymph node detected $(48,63.2 \%)$, but cystic degeneration and necrosis $(18,23.7 \%)$, surrounding organ invasion $(2,2.6 \%)$ and distant metastasis $(5,6.6 \%)$ were less common.

The disease-free survival (DFS) analysis was performed and the 1-, 3- and 5-year DFS rates were $80.7 \%, 72.0 \%$ and $66.1 \%$, respectively. Similarly, the 1-, 3- and 5-year OS rates were $89.2 \%, 68.7 \%$ and $56.7 \%$, respectively. Moreover, univariate survival analysis revealed that diagnostic variables including TNM stage and pathological grade had prognostic significance for DFS and OS $(\mathrm{P}<0.05)$. Towards imaging features, the absence of serosa involvement, heterogeneous enhancement and lymph node metastasis had protective prognostic value (Figure 1; $\mathrm{P}<0.05$ ), however, the lesion site, tumor size, growth pattern, tumor margin integrality, ulceration surrounding organ invasion and distant metastasis didn't show the same effect $(\mathrm{P}>0.05)$. Besides, the presence of cystic degeneration and necrosis had a significantly negative prognostic value for DFS (Figure $1 C ; \mathrm{P}<0.05$ ), but for OS, it only had nearly significant trend (Figure $1 G ; \mathrm{P}=0.053$ ). Then we assumed other variables including treatment, staging and grading might confuse the real findings. Among complex therapeutic choices in our patients, whether radical resection and adjuvant chemotherapy were performed were the only 
Table 2 Univariate analysis of CT characteristics with pathological classifications

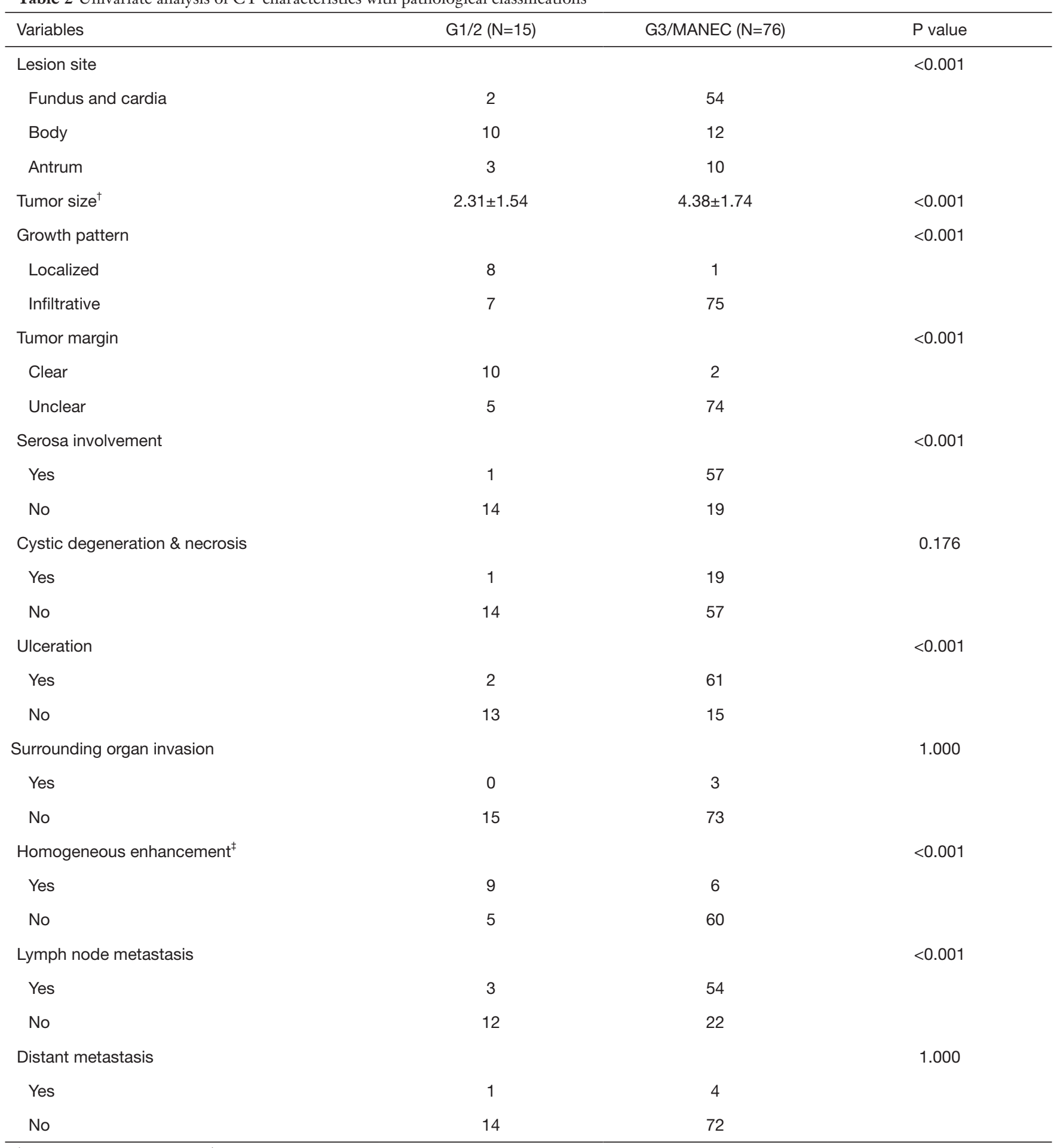

\footnotetext{
${ }^{\dagger}$, the maximum diameter. ${ }^{\ddagger}, 11$ patients lacked enhanced CT images.
} 
Table 3 Sensitivities and specificities of significant CT discriminators for pathological grades

\begin{tabular}{lcc}
\hline Variables & Sensitivity (\%) & Specificity (\%) \\
\hline Tumor size $>3.0 \mathrm{~cm}$ & 76.3 & 80.0 \\
Infiltrative growth & 98.7 & 53.3 \\
Unclear margin & 97.4 & 66.7 \\
Serosa involvement & 75.0 & 93.3 \\
Ulceration & 80.3 & 86.7 \\
Heterogeneous enhancement & 90.9 & 64.3 \\
Lymph node metastasis & 71.1 & 80.0 \\
\hline
\end{tabular}

Table 4 CT characteristics comparison in G3 patients based on Ki-67 index (N=47, 7 patients lost their Ki-67 data)

\begin{tabular}{lccc}
\hline Variables & Ki-67 $\leq 55 \%(\mathrm{~N}=17)$ & Ki-67 $>55 \%(\mathrm{~N}=30)$ & \\
\hline Serosa involvement & 10 & 28 & 0.007 \\
Yes & 7 & 2 & 0.004 \\
No & & & 27 \\
Lymph node metastasis & 8 & 3 \\
Yes & 9 & & \\
No & 9 & & \\
\hline
\end{tabular}

two variables that significantly affected OS and DFS $(\mathrm{P}<0.05)$. Then multivariate COX regression analyses were implemented and variables that were selected including pathological grade, TNM stage, serosa involvement, homogeneous enhancement, cystic degeneration and necrosis, lymph node metastasis detected in the $\mathrm{CT}$, radical resection and adjuvant chemotherapy. Interestingly, serosa involvement was found to be the independent risk factor associated with DFS (HR 3.949; $\mathrm{P}=0.031$ ), but for OS, lymph node metastasis was the only significant risk factor (HR 4.254; $\mathrm{P}=0.021$ ) (Figure 2).

Besides, to further investigate the heterogeneity in G3 g-NECs, the DFS analysis was performed in subgroups of G3 g-NECs with high or low Ki-67 index (55\% as the cutoff point) and it showed a great significance, with the 1 -year DFS rates of $61.7 \%$ and $92.3 \%$, respectively (Figure 3; $\mathrm{P}<0.05$ ). However, unlike DFS, OS comparation between two subgroups had no significantly difference $(\mathrm{P}>0.05)$.

\section{Discussion}

The $\mathrm{g}-\mathrm{NEN}$ is a type of rare and heterogeneous disease which is derived from the diffuse neuroendocrine system in the stomach. Though the incidence is increasing $(6,7)$, early diagnosis and treatment are still difficult due to its nonspecific clinical manifestation and the lack of early detection means. Clarifying the diagnosis of a g-NEN mostly depends on histopathology and the pathological grading is the most important indicator that affect the treatment choice and prognosis (18). However, the acquisition of the pathologic specimen mainly relies on surgical resection while the endoscopic biopsy can only obtain a small amount of tumor tissues which cannot reveal the true pathological diagnosis due to its heterogeneity. As a conventional and noninvasive examination method in clinical practice, though CT cannot replace the value of pathological examination in diagnosis, CT manifestations could evaluate the invasiveness of a lesion and help early diagnosis before the surgery. Meanwhile, CT features have the potential to indicate disease prognosis, which helps formulate appropriate therapeutic strategies at the early stage. Nevertheless, few researches evaluated the value of CT images on predicting pathological grades and prognosis in $\mathrm{g}$-NENs. In the present study, we recruited 98 patients with $\mathrm{g}-\mathrm{NENs}$, with most of them were g-NECs. To our 
Page 8 of 12

Yan et al. Value of CT in pathologic classification and prognosis prediction of gastric NETs

Table 5 Univariate analysis of CT characteristics with survival outcomes

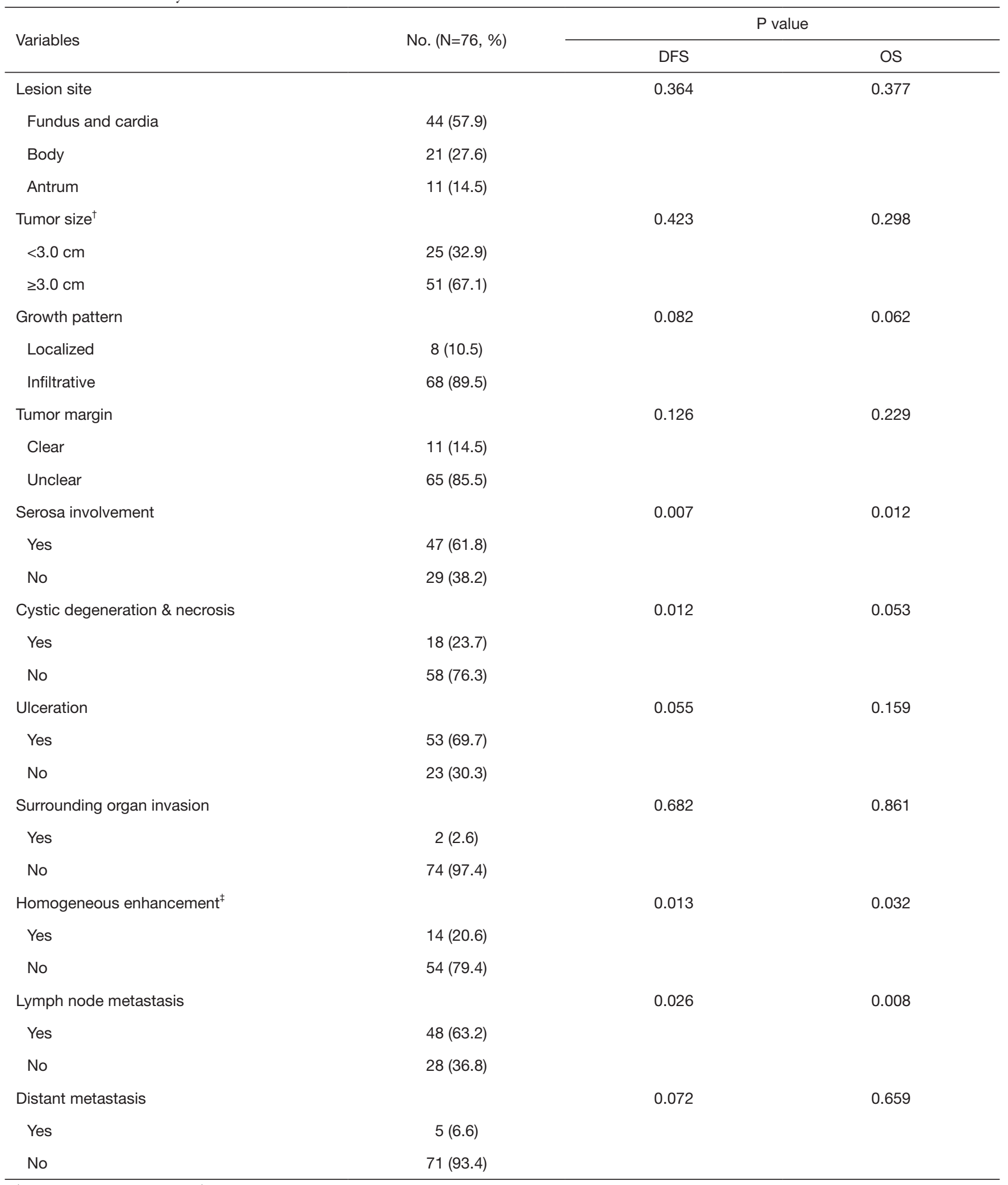

\footnotetext{
${ }^{\dagger}$, the maximum diameter. ${ }^{\ddagger}, 8$ patients lacked enhanced CT images for survival analysis. DFS, disease-free survival; OS, overall survival.
} 
A

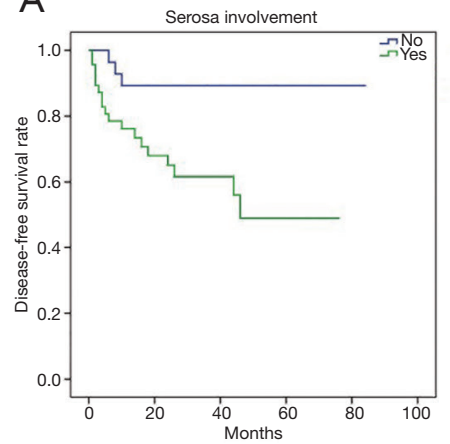

E

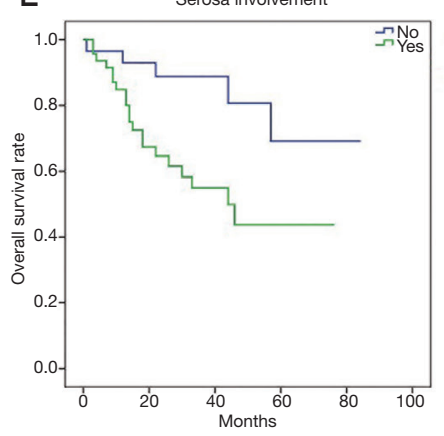

B

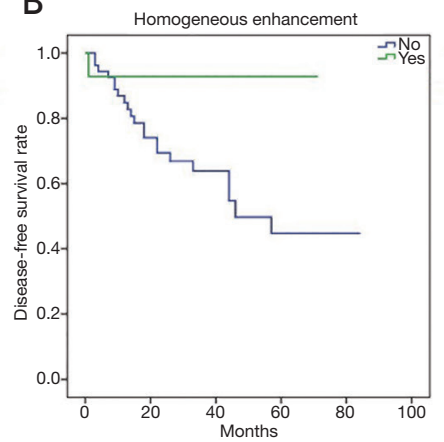

$\mathrm{F}$

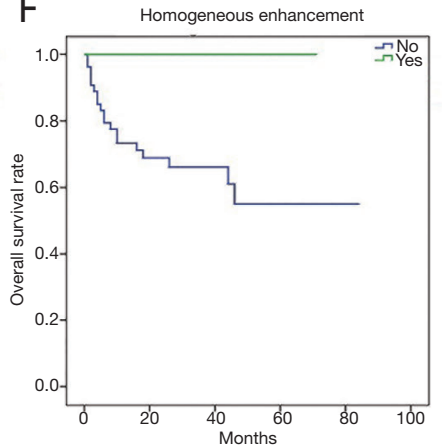

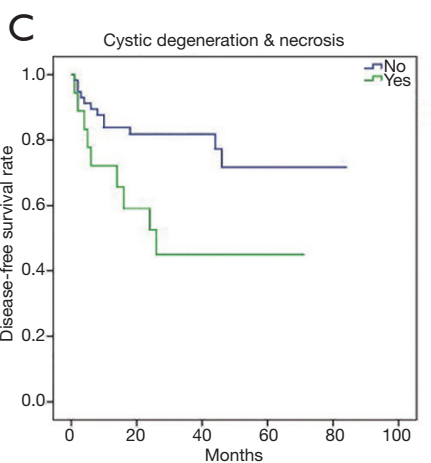

G

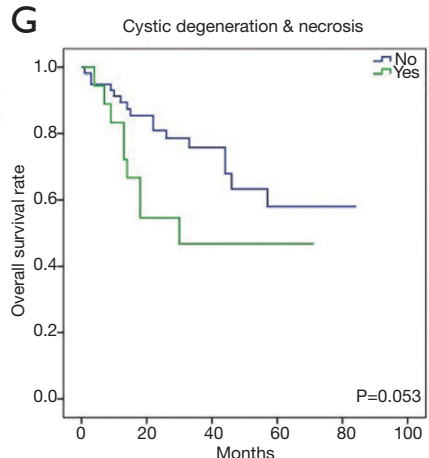

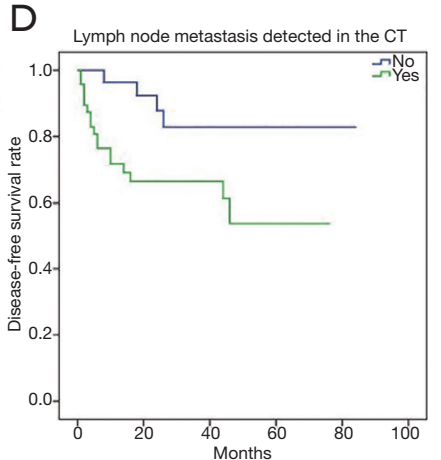

H Lymph node metastasis detected in the CT

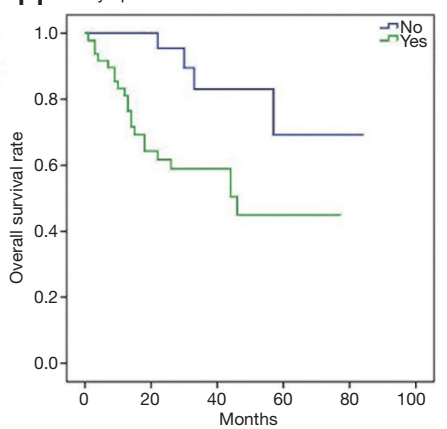

Figure 1 Kaplan-Meier curves for disease-free survival and overall survival based on serosa involvement (A,E), homogeneous enhancement $(\mathrm{B}, \mathrm{F})$, cystic degeneration \& necrosis $(\mathrm{C}, \mathrm{G})$ and lymph node metastasis $(\mathrm{D}, \mathrm{H})$.

A

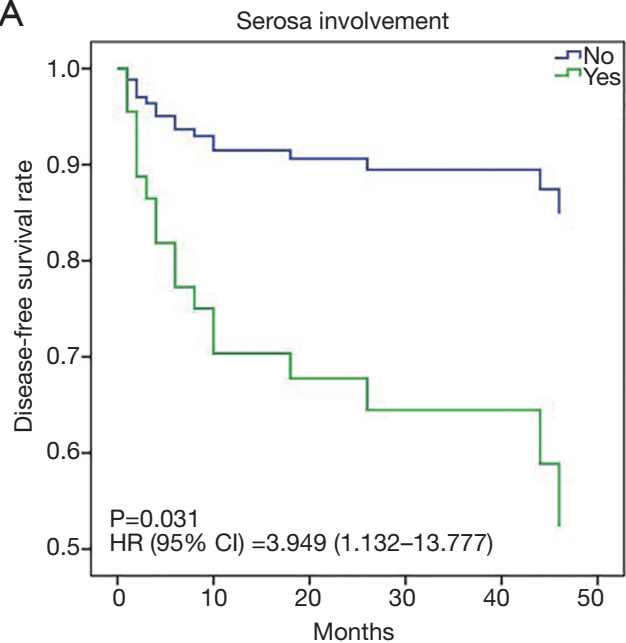

B

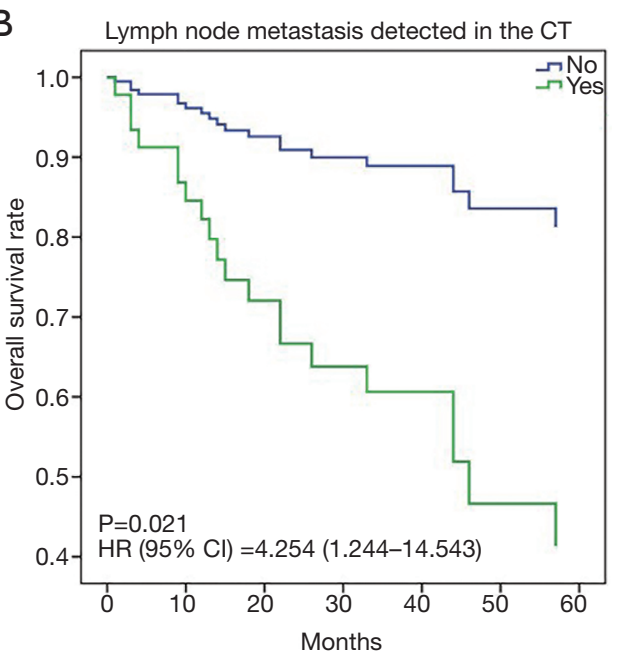

Figure 2 COX regression analysis showed serosa involvement (A) and lymph node metastasis (B) were independent risk factor for DFS and OS, respectively. DFS, disease-free survival; OS, overall survival.

knowledge, this is the first study that comprehensively depicts the correlation of CT findings with pathological grades and prognosis within a relatively large cohort.

The patients demographic characteristics, including a male predominance (1.68:1) and an average age of 60 years old (range, 28-83 years), were in consistent with previous studies $(19,20)$, but date from SEER database showed a reasonable difference (6). The majority of lesions were 


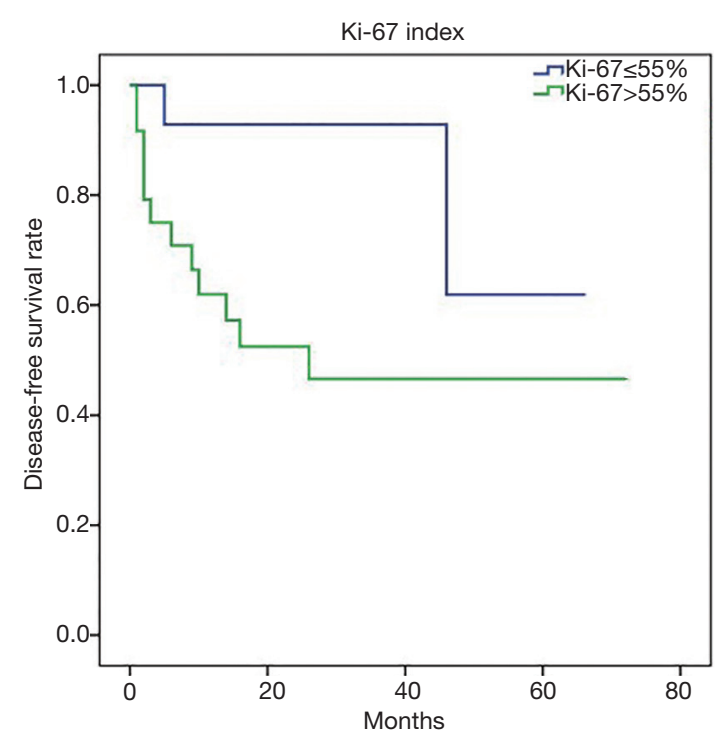

Figure 3 Kaplan-Meier curves for disease-free survival in G3 patients based on $\mathrm{Ki}-67$ index.

detected in the gastric fundus and cardia. Besides, g-NECs usually arose in the gastric fundus and cardia, with a statistic significance $(\mathrm{P}<0.01)$, indicated that the lesion location could predict its malignancy, to a degree (19). The tumor size also shows the same tendency as the pathological grade increases. Kim et al. (21) and Feng et al. (22) provided the optimal cut-off tumor size of 3.05 and $4.0 \mathrm{~cm}$ to distinguish poor-differentiated NETs from well-differentiated NETs, respectively, which were in agreement with our data. By ROC analysis, the cut-off tumor maximum diameter of our 98 patients was $3.0 \mathrm{~cm}$, with an AUC, sensitivity, and specificity of $0.836,76.3 \%$ and $80.0 \%$, respectively, demonstrated a remarkable value of predicting tumor malignancy before surgeries.

Based on our analysis, other imaging characteristics that could suggest aggressive lesions, i.e., g-NECs or g-MANECs, included infiltrative growth pattern, unclear tumor margin, serosa involvement, ulceration, heterogeneous enhancement and lymph node metastasis. Theoretically, as a malignant lesion, g-NEC would be more likely to grow infiltratively and invasively, more possible to have ulcer and metastasis as well, which explained our results (23). However, no significant distinction was showed for variables including cystic degeneration, necrosis, surrounding organ invasion and distant metastasis, which might be related to the fairly small sample size. Furthermore, researches revealed that tissues of pancreatic NECs have low micro-vessel density and are more likely to have carcinoma cell embolus, which would affect intratumoral blood flow and heterogenous enhancement would appear (24). Forty-five patients in our study were discovered carcinoma cell embolus in their pathologic specimens, while 36 of them had heterogenous enhancement during enhanced CT. Therefore, we assumed it might partly explain the reason of relevance between heterogenous enhancement and NECs. Besides, although lymph node metastasis is associated with high pathological grade, metastatic lymph nodes still appear in G1 and G2 digestive NETs $(25,26)$. Hence, regardless of tumor size and pathological grade, the possibility of lymph node metastasis should be aware.

The prognosis of $g$-NEN, especially type III g-NEN, is rather unfavorable. According to the SEER database, the 5-year OS of g-NENs was $53.7 \%$ (9), while a clinicopathological study in China revealed the 5-year OS in Chinese was nearly 60\% (8). Besides, another study from SEER database found the OS for $g$-NENs improved over decades, reflecting the improvement of early diagnosis and treatment (6). The 5-year DFS and OS in our cohort were $66.1 \%$ and $56.7 \%$, respectively, which coincided with previous analyses. Our study also found CT features could predict DFS and OS in patients with g-NENs. Several distinct characteristics showed significantly prognostic value for DFS and OS, such as serosa involvement, enhancement pattern and lymph node metastasis. This result is consistent with previous studies of GEP NENs. Multiple researches demonstrated the correlation of metastatic lymph nodes with prognosis (27-29); moreover, the number, ratio and site of metastatic lymph nodes were also of prognostic significance (27), which indicated the importance of subdividing patients with g-NENs according to the situation of lymph node metastasis. Besides, previously we mentioned the potential reason and diagnostic value of heterogenous enhancement, while survival analysis supported the importance of it as a prognostic variable. Researches on pancreatic NENs had revealed the relationship of the degree of enhancement with prognosis, which was explained by the high proliferative index and associated low microvessel density in NECs $(24,30)$. However, study on the enhancement pattern in g-NENs is lacking and the correlation between enhancement pattern and prognosis of $\mathrm{g}$-NENs needs to be further investigated.

To eliminate the impact of confounding factors for prognosis analysis, multivariate COX regression analyses were performed, which demonstrated that serosa involvement and lymph node metastasis were the only independent risk factor for DFS and OS, respectively. This finding reveals that despite of pathological grading, TNM 
staging and diverse therapeutic strategies, CT characteristics still have remarkable prognostic value for $\mathrm{g}$-NENs.

Furthermore, the G3 g-NENs have been found to be a relatively heterogeneous group concerning therapeutic options and prognosis. As the G3 well-differentiated NET was presented in 2017 WHO classification of pancreatic NEN (31), G3 GEP-NEN was also proved better to subdivide into G3 NEC and G3 NET, based on morphologic differentiation, Ki-67 index, genetic background and clinical history (3). Therefore, further distinguishing G3 g-NETs from G3 g-NECs is still an unmet need in high-grade GEP-NENs (5) and CT manifestations could be useful to identify. The G3 patients in our cohort were roughly parted according to Ki-67 index. It turned out that G3 patients with Ki-67 index $>55 \%$ were significantly more likely to have serosa involved and metastatic lymph nodes, which indicates its invasiveness. Accordingly, the 1-year DFS of G3 NECs with Ki-67 >55\% was significantly worse than the opposite group $(61.7 \%$ s. $92.3 \% ; \mathrm{P}<0.05)$. Hence, as a preliminary result, we found the necessity to define a new subgroup of gastric NEN G3 and to precisely distinguish G3 g-NET with G3 g-NEC; moreover, CT characteristics could help make the final diagnosis by revealing its tendency of invasiveness.

This study has several limitations. Firstly, g-NEN is a relatively rare disease and as a single-center study, our case number is limited. As a rare disease, the awareness of standard diagnosis and treatment is lacking in clinical practice for g-NENs, which led to the confusion and imperfection in the original data, such as the lack of necessary examination for clinical diagnosis and the complex therapeutic choices. It reminds us the necessity of strengthening the awareness of the disease, $g-N E N$, and further investigating a better diagnosis and treatment strategy. Secondly, as a tertiary medical center, most patients had already been advanced stages and high pathological grades when administrated to our hospital. Therefore, cases of $g$-NETs were relatively less and selection bias might present. Third, as a retrospective study, standard contrastenhanced CT scans were not performed in all patients, hence the characteristics of the enhancement pattern and blood supply of g-NENs could not be analyzed thoroughly. In conclusion, perspective studies with larger sample size are warranted to better evaluate CT features of g-NEN and its correlation with pathological characteristics and prognosis.

\section{Acknowledgments}

None.

\section{Footnote}

Conflicts of Interest: The authors have no conflicts of interest to declare.

Ethical Statement: The authors are accountable for all aspects of the work in ensuring that questions related to the accuracy or integrity of any part of the work are appropriately investigated and resolved. The study protocol has been approved by the research institute's committee on human research (No. 19/259-2043). Written informed consent was obtained from the patient for publication of this manuscript and any accompanying images.

\section{References}

1. Bosman FT, Carneiro F, Hruban RH, et al. WHO classification of tumours of the digestive system. World Health Organization 2010.

2. Sahani DV, Bonaffini PA, Fernández-Del Castillo C, et al. Gastroenteropancreatic Neuroendocrine Tumors: Role of Imaging in Diagnosis and Management. Radiology 2013;266:38-61.

3. Sorbye H, Baudin E, Perren A. The Problem of HighGrade Gastroenteropancreatic Neuroendocrine Neoplasms: Well-Differentiated Neuroendocrine Tumors, Neuroendocrine Carcinomas, and Beyond. Endocrinol Metab Clin North Am 2018;47:683-98.

4. Tang LH, Basturk O, Sue JJ, et al. A Practical Approach to the Classification of WHO Grade 3 (G3) Welldifferentiated Neuroendocrine Tumor (WD-NET) and Poorly Differentiated Neuroendocrine Carcinoma (PDNEC) of the Pancreas. Am J Surg Pathol 2016;40:1192-202.

5. Sorbye H, Baudin E, Borbath I, et al. Unmet Needs in High-Grade Gastroenteropancreatic Neuroendocrine Neoplasms (WHO G3). Neuroendocrinology 2019;108:54-62.

6. Dasari A, Shen C, Halperin D, et al. Trends in the Incidence, Prevalence, and Survival Outcomes in Patients With Neuroendocrine Tumors in the United States. JAMA Oncol 2017;3:1335-42.

7. Fan JH, Zhang YQ, Shi SS, et al. A nationwide retrospective epidemiological study of gastroenteropancreatic neuroendocrine neoplasms in china. Oncotarget 2017;8:71699-708.

8. Fang C, Wang W, Zhang Y, et al. Clinicopathologic characteristics and prognosis of gastroenteropancreatic neuroendocrine neoplasms: a multicenter study in South China. Chin J Cancer 2017;36:51. 
9. Cao LL, Lu J, Lin JX, et al. Incidence and survival trends for gastric neuroendocrine neoplasms: An analysis of 3523 patients in the SEER database. Eur J Surg Oncol 2018;44:1628-33.

10. Tian FX, Cai YQ, Zhuang LP, et al. Clinicopathological features and prognosis of patients with gastric neuroendocrine tumors: A population-based study. Cancer Med 2018;7:5359-69.

11. Zhang M, Zhao P, Shi X, et al. Clinicopathological features and prognosis of gastroenteropancreatic neuroendocrine neoplasms in a Chinese population: a large, retrospective single-centre study. BMC Endocr Disord 2017;17:39.

12. Garcia-Carbonero R, Sorbye H, Baudin E, et al. ENETS Consensus Guidelines for High-Grade Gastroenteropancreatic Neuroendocrine Tumors and Neuroendocrine Carcinomas. Neuroendocrinology 2016;103:186-94.

13. Sundin A, Arnold R, Baudin E, et al. ENETS Consensus Guidelines for the Standards of Care in Neuroendocrine Tumors: Radiological, Nuclear Medicine \& Hybrid Imaging. Neuroendocrinology 2017;105:212-44.

14. D'Elia F, Zingarelli A, Palli D, et al. Hydro-dynamic CT preoperative staging of gastric cancer: correlation with pathological findings. A prospective study of 107 cases. Eur Radiol 2000;10:1877-85.

15. Saito T, Kurokawa Y, Takiguchi S, et al. Accuracy of multidetector-row CT in diagnosing lymph node metastasis in patients with gastric cancer. Eur Radiol 2015;25:368-74.

16. Rindi G, Kloppel G, Alhman H, et al. TNM staging of foregut (neuro)endocrine tumors: a consensus proposal including a grading system. Virchows Arch 2006;449:395-401.

17. Delle Fave G, Kwekkeboom DJ, Van Cutsem E, et al. ENETS Consensus Guidelines for the management of patients with gastroduodenal neoplasms. Neuroendocrinology 2012;95:74-87.

18. Garcia-Carbonero R, JImenez-Fonseca P, Teulé A, et al. SEOM clinical guidelines for the diagnosis and treatment of gastroenteropancreatic neuroendocrine neoplasms (GEP-NENs) 2014. Clin Transl Oncol 2014;16:1025-34.

19. Liu DJ, Fu XL, Liu W, et al. Clinicopathological, treatment, and prognosis study of 43 gastric neuroendocrine carcinomas. World J Gastroenterol 2017;23:516-24.

20. Liang P, Wang YX, Ren XC, et al. Neuroendocrine carcinoma of the stomach: clinical features and CT findings. Abdom Radiol (NY) 2016;41:19-24.

21. Kim SH, Kim SH, Kim MA, et al. CT differentiation of poorly-differentiated gastric neuroendocrine tumours from well-differentiated neuroendocrine tumours and gastric adenocarcinomas. Eur Radiol 2015;25:1946-57.

22. Feng ST, Luo Y, Chan T, et al. CT evaluation of gastroenteric neuroendocrine tumors: relationship between ct features and the pathologic classification. AJR Am J Roentgenol 2014;203:W260-6.

23. Levy AD, Sobin LH. Gastrointestinal Carcinoids: Imaging Features with Clinicopathologic Comparison. RadioGraphics 2007;27:237-57.

24. d'Assignies G, Couvelard A, Bahrami S, et al. Pancreatic Endocrine Tumors: Tumor Blood Flow Assessed with Perfusion CT Reflects Angiogenesis and Correlates with Prognostic Factors1. Radiology 2009;250:407-16.

25. Xu G, Wang P, Xiao Y, et al. Local resection of rectal neuroendocrine tumor with first clinical manifestation of giant liver metastasis by transanal endoscopic microsurgery: A case report. Medicine (Baltimore) 2017;96:e9153.

26. Jin $\mathrm{K}, \mathrm{Xu} \mathrm{J}$, Chen J, et al. Surgical management for non-functional pancreatic neuroendocrine neoplasms with synchronous liver metastasis: A consensus from the Chinese Study Group for Neuroendocrine Tumors (CSNET). Int J Oncol 2016;49:1991-2000.

27. Tang X, Chen Y, Guo L, et al. Prognostic significance of metastatic lymph node number, ratio and station in gastric neuroendocrine carcinoma. J Gastrointest Surg 2015;19:234-41.

28. Shen C, Chen H, Chen H, et al. Surgical treatment and prognosis of gastric neuroendocrine neoplasms: a singlecenter experience. BMC Gastroenterol 2016;16:111.

29. Yang $Z$, Wang W, Lu J, et al. Gastric Neuroendocrine Tumors (G-Nets): Incidence, Prognosis and Recent Trend Toward Improved Survival. Cell Physiol Biochem 2018;45:389-96.

30. Kim DW, Kim HJ, Kim KW, et al. Prognostic value of CT findings to predict survival outcomes in patients with pancreatic neuroendocrine neoplasms: a single institutional study of 161 patients. Eur Radiol 2016;26:1320-9.

31. Lloyd RV, Osamura RY, Klöppel G, et al. WHO Classification of Tumours of Endocrine Organs 4th Edition. IARC Press, Lyons, France, 2017.

Cite this article as: Yan S, Liu T, Li Y, Zhu Y, Jiang J, Jiang $\mathrm{L}$, Zhao $\mathrm{H}$. Value of computed tomography evaluation in pathologic classification and prognosis prediction of gastric neuroendocrine tumors. Ann Transl Med 2019;7(20):545. doi: 10.21037/atm.2019.09.114 

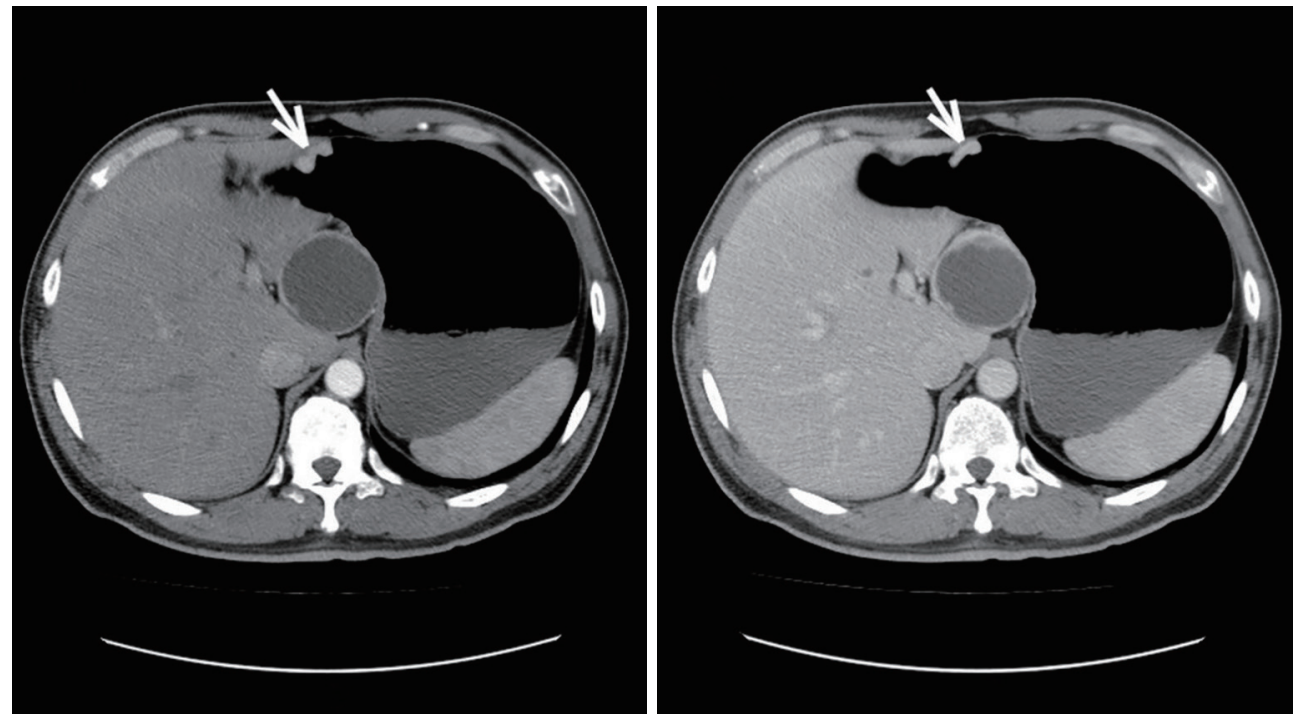

Figure S1 The representative CT image of g-NET. A 46-year-old male diagnosed with gastric neuroendocrine tumor (G1). Enhanced abdominal CT showed a lesion at anterior wall of gastric body (white arrows), with clear margin and significant enhancement during enhanced scan; another solid-cystic lesion at serosa side of lesser curvature of stomach (black arrows), the solid part was enhanced while the cystic part was not.
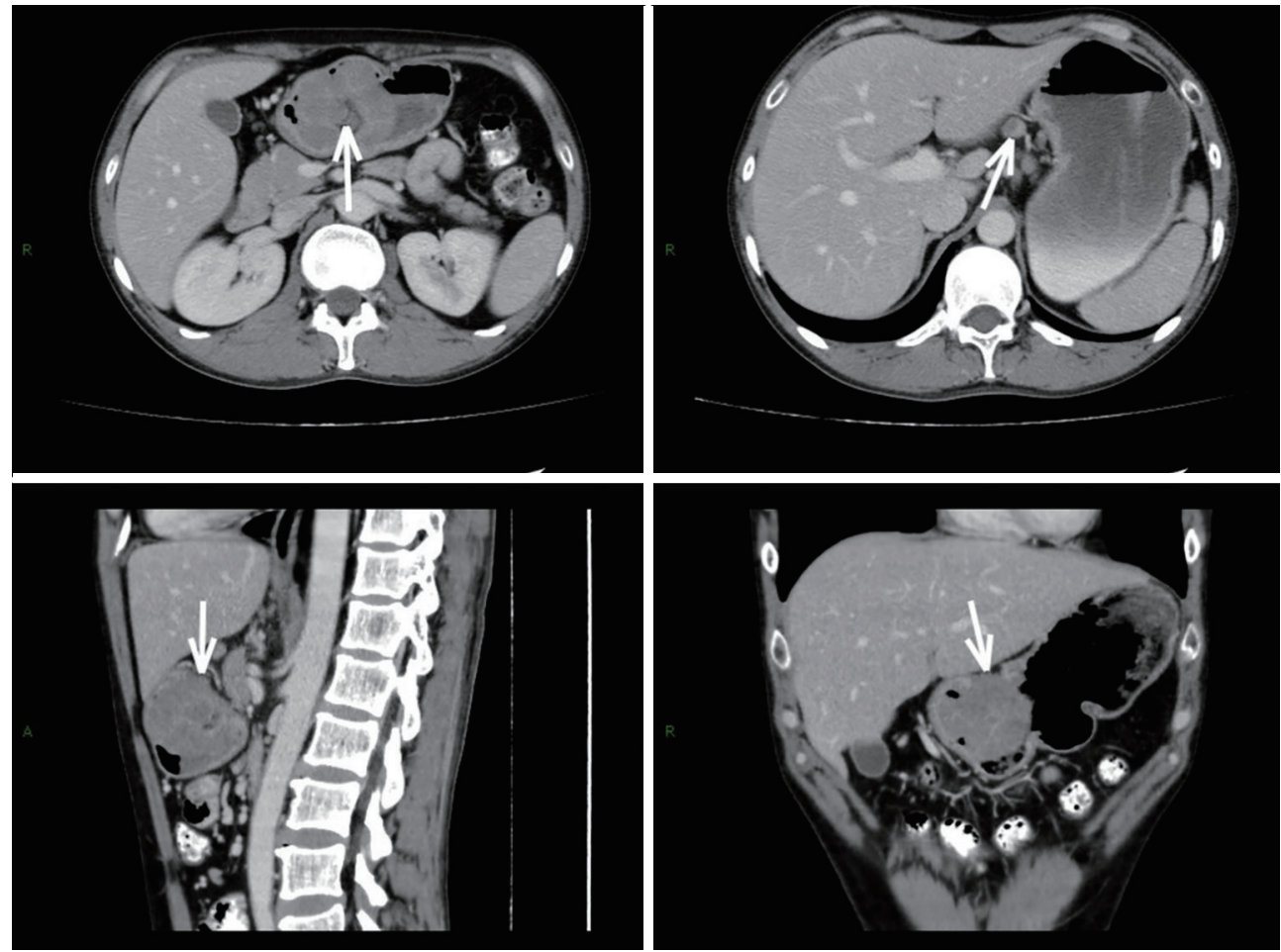

Figure S2 The representative CT image of g-NEC. A 33-year-old male diagnosed with gastric neuroendocrine carcinoma (G3). The enhanced abdominal CT showed an irregular mass at the antrum (white arrows), with unclear margin and rough serosa partly. The lesion had irregular enhancement during enhanced scan. Multiple enlarged lymph nodes were seen at the left side of stomach. 